\title{
Running too far ahead? Towards a broader understanding of mindfulness in organisations
}

\author{
Silke Rupprecht \\ Radboud University \\ Comeniuslaan 4 \\ 6525 HP Nijmegen \\ The Netherlands \\ silke.rupprecht@me.com \\ Wibo Koole* \\ Centrum voor Mindfulness \\ Raadhuisstraat 15 \\ 1016 DB Amsterdam \\ The Netherlands \\ wibokoole@cvm.nl \\ Michael Chaskalson \\ Mindfulness Works \\ 41 St. Andrews Rd. \\ Cambridge CB4 1DH \\ United Kingdom \\ michael@mbsr.co.uk \\ Chris Tamdjidi \\ Kalapa Leadership Academy \\ Genter Strasse 8 \\ 50672 Cologne \\ Germany \\ chris.tamdjidi@kalapaacademy.com
}

Michael West

Lancaster University

Bailrigg

Lancaster LA1 4YX

United Kingdom

m.west@kingsfund.org.uk

*Corresponding author:

Wibo Koole

Centrum voor Mindfulness

Raadhuisstraat 15

1016 DB Amsterdam

The Netherlands

email:wibokoole@cvm.nl 


\section{Declaration of interest}

Silke Rupprecht: none.

Wibo Koole is Director of the Centrum voor Mindfulness, a not-for-profit company that offers mindfulness programs for organizations.

Michael Chaskalson is CEO of Mindfulness Works Ltd., a company that teaches mindfulness in organizational settings.

Chris Tamdjidi is director of Kalapa Institute, a company that teaches mindfulness in organizations.

Michael West: none. 


\begin{abstract}
Current workplace mindfulness research and interventions assume that teaching mindfulness will have beneficial effects for people and organizations. While research shows that mindfulness trainings may increase resilience of working adults, assuming that mindfulness will have independent effects on outcomes at different levels of an organization is not well grounded. We assert that mindfulness training would, however, be beneficial for organizations when tailored to that context and shaped by an understanding of organizational theory and practice. We also envisage mindfulness as a beneficial property of teams, organizations and the individuals who constitute them. To close the evidence gap we propose building multi-level models of mindfulness in organizations, broadening training programs, and developing a novel competency framework for teachers in this context.
\end{abstract}

\title{
Highlights
}

- Mindfulness improves resilience in working adults, but evidence for other work context outcomes such as performance is inconsistent.

- Mindfulness interventions which also focus on team and organizational processes may be more effective than mindfulness alone

- Research should be guided by multilevel models that offer explanations based on interactions between mindfulness and key organizational factors.

- To ensure high quality training for mindfulness in organizations we propose the development of a competency framework for trainers

- Mindful and compassionate leadership should facilitate team and organizational mindfulness.

\section{Research: the evidence gap}

A variety of mindfulness-based trainings (MTs) have been developed for workplaces and for specific target audiences in these - such as leaders. These trainings are either adaptations from MBSR programmes [1,2], or programmes based on different theoretical models such as emotional intelligence [3] or Buddhist philosophy [4-6]. Unlike traditional MTs, workplace MTs vary greatly in length (1 day to 16 weeks) and modes of delivery (apps, webinar, live training) to meet the demands and budgets of organizations [7]. In addition to formal mindfulness practices, a number of informal practices have been developed that foster mindfulness at work and of work - mindful communication, mindful emailing, mindfulness of transitions between tasks and moments of silence $[1,2,5]$. Understandably, practitioners and organizations are interested in research about the potential for MTs to enable employees and leaders to thrive and be effective in the work environment.

Workplace mindfulness research has been largely influenced by MBSR/MBCT modelling - the general approach being that teaching people mindfulness in organizations will have beneficial effects for them and their organizations [8]. There is now consistent evidence that mindfulness-based interventions are effective in reducing stress in working adults $[7,9]$ as well as symptoms of depression and addiction in clinical populations $[10,11]$. To date, $80 \%$ of mindfulness intervention studies in workplaces have focused on stress and resilience as their primary outcomes variables [12] but the overall study quality remains low [7].

Studies about the impact of mindfulness training on workplace-specific outcomes such as leadership, prosocial behaviour at work and performance [8] have produced at best 
preliminary evidence of positive outcomes. Studies investigating mindfulness and leadership, for example, are predominantly correlational [13-15] and found that mindful leadership was associated with humility and authenticity [13], follower wellbeing and citizenship [14] and good quality of leader-follower interactions [15]. But intervention studies investigating the effect of mindfulness on key aspects of leadership are scarce [16]. Mindfulness may be associated with increases in prosocial behaviour at work $[17,18]$, demonstrated by greater empathy and compassion $[19,20]$ and higher interaction quality, but Gebauer and colleagues [21] challenge the notion that MT is related to greater other-orientation by showing that instead of muting the ego it enhanced self-centrality. As a final example there have been inconsistent outcomes regarding the association of mindfulness with performance indicators. Some studies show that mindfulness may be associated with improved individual $[5,22-24]$ and team performance $[25,26]$. Other studies report negative or no effects of state mindfulness or MT on performance indicators $[27,28]$.

Such contradictory outcomes may partly be explained by the overall weak study quality $[7,17]$. Perhaps more salient is the timid and theoretically weak approach to conceptualizing, researching and teaching mindfulness in organizations. The gap between evidence and training practice is reflected in a debate [29] about whether it is ethical to offer mindfulness training as a panacea for the problems of modern workplaces such as excessive workloads, bullying, harassment and punitive supervision.

\section{Putting mindfulness in the context of organizations}

Why should mindfulness practice produce significant changes in workplace experience [30], or lead to better organizational outcomes? It seems unlikely that simply encouraging people to practice mindfulness will change the damaging effects of high performance pressure and dysfunctional leadership. Similarly, can we be sure that introducing mindfulness to working environments characterised by bullying or blaming will make a difference to that culture [31]? For a better understanding of mindfulness in the context of organizations we must develop theories that offer explanations based on interactions between mindfulness and significant workplace factors. Furthermore, it will be necessary to expand our current understanding of mindfulness as an individual-level variable to include mindfulness as a team- and organization-level variable.

A first issue that must be addressed includes what is meant by individual mindfulness in organizations and how (or if) this differs from non-work settings [22]. As Lyddy and Good [32] discuss for example in their inductive model, mindfulness practitioners may develop "being while doing" at work, thus applying mindfulness skills to their respective work environments.

Then, we can conceive of mindfulness practices and skills among multiple team members affecting team and organizational processes and outcomes. These might include inter alia reduced conflict, improved interpersonal relationship, greater awareness of errors or problems in work processes, and improved team and organizational productivity - for which there is preliminary evidence [8].

This perspective should then also take into account how organizational factors might moderate the effects of mindfulness practices on team and organizational outcomes. Thus, the combined effects on the outcomes for teams or organizations of multiple members practising mindfulness will be moderated by the extent to which there is a clear, shared purpose, a good value fit between members and the organization overall and a supportive, compassionate and authentic leadership. 
To broaden our understanding we also propose the conceptualization of mindfulness as a supra-individual level phenomenon. Mindfulness can emerge as a team-level variable in team units based on team experiences [26,33]. A provisional definition of team mindfulness would describe it as collectively paying attention to the team experiences and their underlying objectives, tasks, roles and structures, in a periodically consistent and nonjudgmental way. Extensive research on team reflexivity has demonstrated that through a sustained collective awareness of purpose, performance, processes, climate and problems, teams are better able to ensure effective team functioning [33-37]. Just as one returns to present moment awareness in the practice of individual mindfulness, so mindful teams will repeatedly return their awareness or attention to purpose, performance, processes, climate and problems. This may be mediated by processes such as changes in present moment experiencing and by the adoption of non-judgmental attitudes thus allowing members to become more sensitive to the dynamics of their teams and more capable of adapting team structures and processes [26].

Team mindfulness is thus distinct from individual mindfulness but may be enhanced by individual mindfulness practice. For example, where there is a high level of individual level mindfulness, it would be possible, through training, to encourage team members to develop and sustain awareness of key team elements. Such sustained attention on these core components of team (and organizational) functioning will increase the likelihood of team and organizational effectiveness. This calls for a clear conceptualization of the interaction between collective individual mindfulness and team (or organizational) mindfulness which would reflect the role of organizational purpose, performance and processes (leadership, decision-making, conflict management, people management) as well as culture, climate and problems (excessive workload, staff shortages, discrimination, conflict) [38]. Thus interventions that focus both on individual mindfulness and on increasing team member awareness of core team (or organizational) characteristics will have more powerful effects than their separate contributions.

Finally, theory might also focus on how mindful and compassionate leadership can create the conditions for effective team working and innovation [39]. There is evidence that compassionate leadership creates conditions for altruism and intrinsic motivation, for risk taking, for speaking about errors, concerns and problems, for developing improved ways of doing things, and for creating a climate of optimism, efficacy and cohesion in teams [40].

All in all, we propose the development of a multi-level model of mindfulness in organizations [41]. This leads to a vision of mindfulness also as a property of teams and organizations as a whole, which differs from Weick et al.'s [42] depiction of organizational mindfulness as (only) a set of practices [43].

\section{The training practice: broader programs and teacher qualifications}

Currently, mindfulness training in organizations focuses on teaching mindfulness to individuals (both employees and leaders). Given that our conceptualizations of mindfulness in organizations are credible, we expect that in addition to developing mindfulness in individuals the need for training programs focusing on mindfulness of team and organizational processes will grow. This means including interpersonal habits, both of individuals and teams and the related group dynamics, in the content of the training programs. Although such a shift might happen unconsciously (e.g. one study showed increased awareness of workplace stressors following MBSR training [44]) leaders and 
employees might be guided in a process so as to become curious about improving team and organizational effectiveness as part of the training [33].

Teacher qualification is a core factor in the success of delivering effective mindfulness trainings in organizations. In developing such qualifications the current focus is on translating the well-formulated MBSR-based standards into the workplace context [45]. For teaching mindfulness in organizations qualifications need to be broadened to having a sound understanding of the possible links between mindfulness practice and team and organizational dynamics.

This calls for the development of a competency framework for teachers of mindfulness in organizations which encompasses both mindfulness teaching skills and the knowledge and skills to apply mindfulness in teams and organizations. Thus, if the goal is to improve team effectiveness in an organization, the combined qualifications of an experienced mindfulness trainer and that of a team leader or coach will be needed. If the goal is stress reduction, a mindfulness intervention may be preceded by an investigation of the factors causing stress in that specific work environment (such as workload, bullying, harassment, discrimination, role ambiguity) and an evaluation of whether mindfulness interventions are sufficient without other organizationally contextualised interventions.

Supporting the development of team and organizational mindfulness presupposes having sufficient knowledge of research into team and organizational functioning and the effective training practices that are based upon that. Then teachers will be equipped to do more than simply teach individual mindfulness practices in organizations. They can couple this with interventions which increase collective awareness of team/organizational purpose, processes, climate, problems etc. to achieve desired individual, team and organizational outcomes.

\section{Conclusions}

Mindfulness in organizations is not only an individual property but also a property of teams and the organization itself. For example, based on their shared understanding of team effectiveness, team members may apply mindfulness to team processes thereby improving team performance and individual well-being. Compassionate and mindful leadership may be particularly potent and salient in guiding this, given the influence of leaders in teams.

Future research should focus on gaining a deeper understanding of the potential and limits of training individuals in mindfulness in a workplace context. To start, qualitative and correlational research might help us to ground new theories and models. Intervention studies could then include active control conditions such as team effectiveness coaching and compare the differential impact of coaching vs mindfulness in a team setting along with a combination of both. In general, more high-quality studies employing longitudinal and multilevel designs that account for the nested structure of organizations (individuals, teams, departments, organizations) will enrich our understanding. Furthermore, potential mediators and moderators on each level of the organization (e.g., supervisory support, role clarity, clarity of team purpose and objectives, organizational culture) should be taken into consideration $[26,46]$. This also implies recruiting whole teams or organizations rather than a self-selected sample of working people interested in practising mindfulness.

Mindfulness training organizations may need to broaden their approach and to develop programs that integrate mindfulness at team and organizational level. Research into team and organizational effectiveness must be integrated into our thinking about how to apply mindfulness in organizations. Trainings with individuals, team and whole organizations 
should apply a competency framework rooted in a comprehensive understanding and embodiment of mindfulness in an organizational context.

Given the evidence gap and the need to broaden our understanding of mindfulness in organizations the biggest contribution perhaps of trainers and researchers to mindfulness in organizations could be the humble acknowledgement that we only know so much. And that offering training individuals in mindfulness as a panacea for modern workplace problems will not do it. Only in this way can mindfulness in organizations live up to its promise and avoid becoming an empty signifier that can be used to sell anything [47].

\section{Acknowledgements}

This research did not receive any specific grant from funding agencies in the public, commercial, or not-for-profit sectors.

\section{References}

[1] M. Chaskalson, The mindful workplace: Developing resilient individuals and resonant organizations with MBSR, Wiley-Blackwell, Chichester, West Sussex ; Malden, MA, 2011.

[2] W. Koole, Mindful leadership: effective tools to help you focus and succeed, Warden Press, Amsterdam, 2014.

[3] C.-M. Tan, Search inside yourself: The unexpected path to achieving success, happiness (and world peace), HarperOne, New York, 2012.

[4] E. Shonin, W. Van Gordon, T.J. Dunn, N.N. Singh, M.D. Griffiths, Meditation Awareness Training (MAT) for Work-related Wellbeing and Job Performance: A Randomised Controlled Trial, Int. J. Ment. Health Addict. 12 (2014) 806-823. doi:10.1007/s11469-014-9513-2.

[5] W. Kersemaekers, S. Rupprecht, M. Wittmann, C. Tamdjidi, P. Falke, R. Donders, A. Speckens, N. Kohls, A workplace mindfulness intervention may be associated with improved psychological well-being and productivity. A preliminary field study in a company setting, Front. Psychol. 9 (2018). doi:10.3389/fpsyg.2018.00195.

[6] R. Hougaard, G. Coutts, One second ahead: enhance your performance at work with mindfulness, Palgrave Macmillan, Houndmills, Basingstoke, Hampshire : New York, NY, 2016.

[7]* T. Lomas, J.C. Medina, I. Ivtzan, S. Rupprecht, R. Hart, F.J. Eiroa-Orosa, The impact of mindfulness on well-being and performance in the workplace: an inclusive systematic review of the empirical literature, Eur. J. Work Organ. Psychol. (2017) 1-22. doi:10.1080/1359432X.2017.1308924.

This systematic review of mindfulness studies set in workplaces showed the great heterogeneity in study quality, samples, training types and outcome variables.

[8]* D.J. Good, C.J. Lyddy, T.M. Glomb, J.E. Bono, K.W. Brown, M.K. Duffy, R.A. Baer, J.A. Brewer, S.W. Lazar, Contemplating mindfulness at work: An integrative review, J. Manag. 42 (2016) 114-142. doi:10.1177/0149206315617003.

This review summarized the assumed potential of mindfulness at work by drawing from evidence of mindfulness research.

[9] A. Chiesa, A. Serretti, Mindfulness-Based Stress Reduction for stress management in healthy people: A review and meta-analysis, J. Altern. Complement. Med. 15 (2009) 593600. doi:10.1089/acm.2008.0495. 
[10] S.B. Goldberg, R.P. Tucker, P.A. Greene, R.J. Davidson, B.E. Wampold, D.J. Kearney, T.L. Simpson, Mindfulness-based interventions for psychiatric disorders: A systematic review and meta-analysis, Clin. Psychol. Rev. 59 (2018) 52-60.

doi:10.1016/j.cpr.2017.10.011.

[11] W. Kuyken, F.C. Warren, R.S. Taylor, B. Whalley, C. Crane, G. Bondolfi, R. Hayes, M. Huijbers, H. Ma, S. Schweizer, Z. Segal, A. Speckens, J.D. Teasdale, K. Van Heeringen, M. Williams, S. Byford, R. Byng, T. Dalgleish, Efficacy of Mindfulness-Based Cognitive Therapy in Prevention of Depressive Relapse: An Individual Patient Data Meta-analysis From Randomized Trials, JAMA Psychiatry. (2016). doi:10.1001/jamapsychiatry.2016.0076.

[12] L.T. Eby, T.D. Allen, K.M. Conley, R.L. Williamson, T.G. Henderson, V.S. Mancini, Mindfulness-based training interventions for employees: A qualitative review of the literature, Hum. Resour. Manag. Rev. (2017) 1-23. doi:10.1016/j.hrmr.2017.03.004.

[13] A. Pircher Verdorfer, Examining mindfulness and its relations to humility, motivation to lead, and actual servant leadership behaviors, Mindfulness. 7 (2016) 950-961.

doi:10.1007/s12671-016-0534-8.

[14]* J. Reb, J. Narayanan, S. Chaturvedi, Leading mindfully: Two studies on the influence of supervisor trait mindfulness on employee well-being and performance, Mindfulness. 5 (2014) 36-45. doi:10.1007/s12671-012-0144-z.

This study developed and tested a model that links leader mindfulness with employee outcomes.

[15] J. Reb, S. Chaturvedi, J. Narayanan, R.S. Kudesia, Leader mindfulness and employee performance: a sequential mediation model of LMX quality, interpersonal justice, and employee stress, J. Bus. Ethics. (2018). doi:10.1007/s10551-018-3927-x.

[16] W. Brendel, S. Hankerson, S. Byun, B. Cunningham, Cultivating leadership Dharma: Measuring the impact of regular mindfulness practice on creativity, resilience, tolerance for ambiguity, anxiety and stress, J. Manag. Dev. 35 (2016) 1056-1078. doi:10.1108/JMD-092015-0127.

[17]* U. Kreplin, M. Farias, I.A. Brazil, The limited prosocial effects of meditation: A systematic review and meta-analysis, Sci. Rep. 8 (2018) 1-10. doi:10.1038/s41598-01820299-z.

* This meta-analysis on mindfulness and prosociality also identified potential biases in mindfulness research.

[18] J.N. Donald, B.K. Sahdra, B. Van Zanden, J.J. Duineveld, P.W.B. Atkins, S.L. Marshall, J. Ciarrochi, Does your mindfulness benefit others? A systematic review and metaanalysis of the link between mindfulness and prosocial behaviour, Br. J. Psychol. (2018). doi:10.1111/bjop.12338.

[19] M.S. Krasner, R.M. Epstein, H.B. Beckman, A.L. Suchman, B. Chapman, C.J. Mooney, T.E. Quill, Association of an Educational Program in Mindful Communication With Burnout, Empathy, and Attitudes Among Primary Care Physicians, JAMA. 302 (2009) 1284. doi:10.1001/jama.2009.1384.

[20] S.L. Shapiro, G.E. Schwartz, G. Bonner, Effects of Mindfulness-Based Stress Reduction on medical and premedical students, J. Behav. Med. 21 (1998) 581-599. doi:10.1023/A:1018700829825.

[21]* J.E. Gebauer, A.D. Nehrlich, D. Stahlberg, C. Sedikides, A. Hackenschmidt, D. Schick, C.A. Stegmaier, C.C. Windfelder, A. Bruk, J. Mander, Mind-Body Practices and the Self: Yoga and Meditation Do Not Quiet the Ego but Instead Boost Self-Enhancement, Psychol. Sci. (2018) 095679761876462. doi:10.1177/0956797618764621. 
This study showed that MT (and Yoga) increased self-enhancement meditated by selfcentrality.

[22] E. Dane, B.J. Brummel, Examining workplace mindfulness and its relations to job performance and turnover intention, Hum. Relat. 67 (2014) 105-128.

doi: $10.1177 / 0018726713487753$.

[23] M.D. Mrazek, M.S. Franklin, D.T. Phillips, B. Baird, J.W. Schooler, Mindfulness Training Improves Working Memory Capacity and GRE Performance While Reducing Mind Wandering, Psychol. Sci. 24 (2013) 776-781.

[24] U.W. Weger, N. Hooper, B.P. Meier, T. Hopthrow, Mindful maths: Reducing the impact of stereotype threat through a mindfulness exercise, Conscious. Cogn. 21 (2012) 471475. doi:10.1016/j.concog.2011.10.011.

[25] P. den Heijer, W. Koole, C.J. Stettina, Don't Forget to Breathe: A Controlled Trial of Mindfulness Practices in Agile Project Teams, in: H. Baumeister, H. Lichter, M. Riebisch (Eds.), Agile Process. Softw. Eng. Extreme Program., Springer International Publishing, Cham, 2017: pp. 103-118. doi:10.1007/978-3-319-57633-6_7.

[26]* L. Yu, M. Zellmer-Bruhn, Introducing team mindfulness and considering its safeguard role against conflict transformation and social undermining, Acad. Manage. J. 61 (2018) 324347. doi:10.5465/amj.2016.0094.

This study developed an instrument to assess team mindfulness and shows that this new construct was associated with less relationship conflict in teams.

[27]* A.C. Hafenbrack, K.D. Vohs, Mindfulness meditation impairs task motivation but not performance, Organ. Behav. Hum. Decis. Process. 147 (2018) 1-15.

doi:10.1016/j.obhdp.2018.05.001.

This experimental study showed that induced state mindfulness decreased motivation to perform a task, even it was framed as pleasant, but did not affect task performance.

[28]* J. van Berkel, C.R.L. Boot, K.I. Proper, P.M. Bongers, A.J. van der Beek, Effectiveness of a Worksite Mindfulness-Related Multi-Component Health Promotion Intervention on Work Engagement and Mental Health: Results of a Randomized Controlled Trial, PLoS ONE. 9 (2014) e84118. doi:10.1371/journal.pone.0084118.

This high-quality study showed that a MT at a worksite was not effective in increasing work engagement and mental health six months post programme completion.

[29] Z. Walsh, E. Ng, R.E. Purser, The promise and perils of corporate mindfulness, in: D. Knights, C. Mabey (Eds.), Leadersh. Matters Find. Voice Connect. Mean. 21st Century, Routledge, S.1., 2017: pp. 1-14.

[30] B. Schneider, V. Gonzalez-Roma, C. Ostroff, M.A. West, Organizational climate and culture: Reflections on the history of the constructs in the Journal of Applied Psychology., J. Appl. Psychol. 102 (2017) 468-482. doi:10.1037/ap10000090.

[31] S. Einarsen, Bullying and harassment in the workplace developments in theory, research, and practice., CRC Press, Boca Raton, 2011.

[32] C.J. Lyddy, D.J. Good, Being While Doing: An Inductive Model of Mindfulness at Work, Front. Psychol. 7 (2017). doi:10.3389/fpsyg.2016.02060.

[33] M.C. Schippers, A.C. Edmondson, M.A. West, Team reflexivity as an antidote to team information-processing failures, Small Group Res. 45 (2014) 731-769. 
doi:10.1177/1046496414553473.

[34] J. Lyubovnikova, M.A. West, J.F. Dawson, M.R. Carter, 24-Karat or fool's gold? Consequences of real team and co-acting group membership in healthcare organizations, Eur. J. Work Organ. Psychol. 24 (2015) 929-950. doi:10.1080/1359432X.2014.992421.

[35] E. Salas, M.L. Shuffler, A.L. Thayer, W.L. Bedwell, E.H. Lazzara, Understanding and Improving teamwork in organizations: A scientifically based practical guide, Hum. Resour. Manage. 54 (2015) 599-622. doi:10.1002/hrm.21628.

[36] M.A. West, Effective teamwork: practical lessons from organizational research, 3rd ed, BPS Blackwell, Malden, Ma, 2012.

[37] M.C. Schippers, A.C. Edmondson, M.A. West, Team reflexivity, in: L. Argote, J. Levine (Eds.), Oxf. Handb. Group Organ. Learn., Oxford University Press, Oxford, in press.

[38] M.A. West, J. Lyubovnikova, R. Eckert, J.-L. Denis, Collective leadership for cultures of high quality health care, J. Organ. Eff. People Perform. 1 (2014) 240-260.

doi:10.1108/JOEPP-07-2014-0039.

[39]* M.A. West, R. Chowla, Compassionate leadership for compassionate health care, in:

P. Gilbert (Ed.), Compassion Concepts Res. Appl., Routledge, London, 2017: pp. 237-57.

This book chapter defined and illustrated how compassionate leadership creates the necessary conditions for a high-quality and innovative culture in health care

[40] M.A. West, R. Eckert, B. Collins, R. Chowla, Caring to change: how compassionate leadership can stimulate innovation in health care, The King's Fund, London, 2017.

[41] K.J. Klein, H. Tosi, A.A. Cannella, Multilevel theory building: Benefits, barriers, and new developments, Acad. Manage. Rev. 24 (1999) 248-253. doi:10.5465/amr.1999.1893934.

[42] K.E. Weick, K.M. Sutcliffe, D. Obstfeld, Organizing for high reliability: Processes of collective mindfulness, Crisis Manag. 3 (2008) 31-66.

[43]* K.M. Sutcliffe, T.J. Vogus, E. Dane, Mindfulness in Organizations: A Cross-Level Review, Annu. Rev. Organ. Psychol. Organ. Behav. 3 (2016) 55-81. doi:10.1146/annurevorgpsych-041015-062531.

The authors reviewed a different conceptualization of organizational mindfulness and their points of contact with individual mindfulness research.

[44] H. Walach, E. Nord, C. Zier, B. Dietz-Waschkowski, S. Kersig, H. Schüpbach, Mindfulness-based stress reduction as a method for personnel development: A pilot evaluation., Int. J. Stress Manag. 14 (2007) 188-198. doi:10.1037/1072-5245.14.2.188.

[45] R.S. Crane, J. Brewer, C. Feldman, J. Kabat-Zinn, S. Santorelli, J.M.G. Williams, W. Kuyken, What defines mindfulness-based programs? The warp and the weft, Psychol. Med. 47 (2017) 990-999. doi:10.1017/S0033291716003317.

[46] J. Reb, J. Narayanan, Z.W. Ho, Mindfulness at Work: Antecedents and Consequences of Employee Awareness and Absent-mindedness, Mindfulness. 6 (2015) 111-122.

doi:10.1007/s12671-013-0236-4.

[47] G. Islam, M. Holm, M. Karjalainen, Sign of the times: Workplace mindfulness as an empty signifier, Organization. (2017) 135050841774064. doi:10.1177/1350508417740643. 